\title{
UNILATERAL RENAL VEIN THROMBOSIS TREATED BY NEPHRECTOMY AND POST-OPERATIVE HEPARIN
}

\author{
BY \\ E. W. PARRY \\ From the Paediatric Unit, County Hospital, Bangor
}

(Received for Publication January 19, 1951)

In the majority of cases renal vein thrombosis is secondary to dehydration, sepsis, or both, and has occurred in enterocolitis, diphtheria, umbilical sepsis, measles, and skin infections. It has been recorded as a sequel to pyelo-nephritis due to a spread from the glomerular to the renal vein. The renal vein may become secondarily involved as a result of thrombophlebitis in the vena cava, the spermatic, or the ovarian veins. This type, however, seems to be confined to adults, and is very rare in infancy.

Both sexes are equally involved. The age incidence is interesting in that $40 \%$ of cases occur in the first two months of life (Abeshouse, 1945).

In the neonatal period the commonest type of lesion is one in which the thrombotic process originates within the renal venous system subsequent to infection with dehydration.

The case described here is one of unilateral renal vein thrombosis diagnosed after observation and treated by nephrectomy.

\section{Case Report}

On March 3, 1950, a boy weighing $10 \mathrm{lb} .6 \mathrm{oz}$. was born at full term; the delivery and pregnancy were normal. The Wassermann reaction was negative, $R h$ positive.

At birth the baby was cyanosed and did not cry well. $\mathrm{He}$ had multiple petechiae of the head, neck, and trunk. There was poor air entry into the lungs. A diagnosis of anoxia due to extensive atelectasis was made and continuous oxygen therapy was administered for three days. Afterwards his condition improved and he was breast fed normally.

On March 29, 1950, the mother insisted on discharging herself and the baby from the hospital. The weight of the baby was now $9 \mathrm{lb}$., and there was some redness and a slight discharge from the cord stump.

On April 4, 1950, the child was readmitted with a history of streaks of blood in the urine and vomiting for three or four days.

When the baby was examined he weighed $8 \mathrm{lb} .6 \mathrm{oz}$., and his temperature was $101 \cdot 2^{\circ} \mathrm{F}$. There was bloodstained mucus on the napkin; this was not mixed with the canary yellow stool, which was normal in appearance.
The child's general condition showed him to be pale and quiet with evidence of dehydration. His chest and heart were normal; his abdomen was normal in appearance and movements. The umbilicus was clean and dry. Palpation revealed a large firm mass extending from the level of the costal margin to the iliac crest on the left side. The mass was perfectly smooth in outline and no notch could be felt. It conformed in outline to a renal swelling and could be displaced from the loin. The mass was obviously painful, and any palpation caused marked distress. No other abnormality could be found on examination. The right kidney was not palpable.

On rectal examination the mass could be felt in front of the rectum at the pelvic brim. No blood was seen on the examining finger. During the examination the child passed a quantity of urine which was preceded by the passage of a blood-stained mucous clot.

Further investigations gave the following results:

A radiograph of the abdomen was negative. A blood count gave R.B.Cs., 4.5 m.; Hb., 95\%, W.B.Cs., 19,400 (neutrophils $63 \%$, lymphocytes $30 \%$ ). Microscopic examination of the urine showed one or two red blood cells, a large excess of leucocytes, coliform organisms, but no casts. On culture only Bact. coli were found.

In view of a large firm tumour of sudden origin in an infant, the differential diagnosis of Wilms' embryoma, infected haematoma of the kidney, or renal vein thrombosis was made. The history of cord infection and vomiting, together with clinical evidence of dehydration, blood-stained and heavily infected urine, and raised temperature, was more in favour of a renal vein thrombosis. The leucocytosis of 19,000 could be within normal limits.

Further blood examinations were made with a view to confirming a diagnosis of thrombosis, and these showed bleeding time, $7 \mathrm{~min}$; clotting time, $2 \frac{1}{2} \mathrm{~min}$.; prothrombin time, $15 \mathrm{sec}$; prothrombin control, $15 \mathrm{sec}$; prothrombin index, $100 \%$.

Although these findings were normal it was thought that the history and clinical findings were sufficient to diagnose a unilateral renal vein thrombosis.

The baby was prepared for exploratory operation.

Pre-operative Treatment. Hartmann's solution, $600 \mathrm{ml}$., was given subcutaneously; 500,000 units of penicillin were given at once and a further 100,000 units six-hourly. After the infusion the general condition 


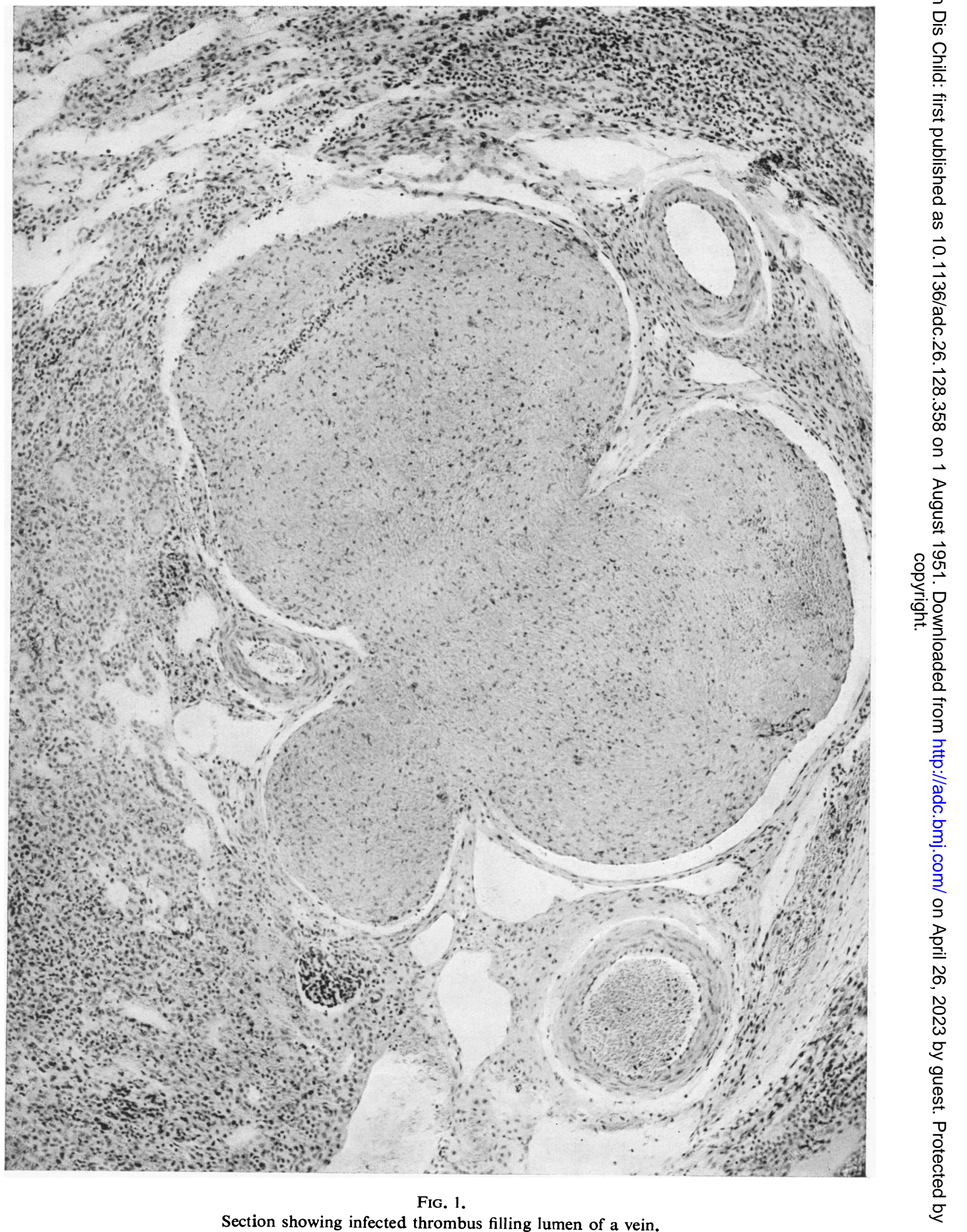


improved considerably and urine was excreted in good quantity.

Operation. The operation was performed on April 5, 1950. Ether vapour and oxygen conducted by catheter under an open mask were used. The baby was placed in a semi-recumbent position with sandbags under the shoulder and pelvis. A left oblique renal incision was used with a right-angled extension upwards from the middle to allow free access to the renal pedicle.

The fascia of Zuckerkandl and fat were thickened and oedematous. Heavily bloodstained fluid was found between the fascia and the kidney, which was enlarged (more than twice the normal size) and haemorrhagic. Examination of the renal pedicle revealed a firm thrombus filling the whole of the renal vein. No thrombus was palpated in the vena cava.

The left kidney was packed off and the peritoneum opened at the anterior end of the wound. A transperitoneal examination of the right kidney showed this to be completely normal in size, shape, and consistency. The peritoneum was closed. The right kidney was normal and the renal output adequate, but the left renal vein was completely thrombosed and the urine heavily infected with Bact. coli, and nephrectomy was decided upon. The pedicle was dissected, and the renal vein ligatured about $\frac{3}{4}$ in. from the vena cava. The renal artery was normal and was ligated separately. The kidney was dissected free and removed. Then the wound was closed in layers with interrupted catgut sutures and the remaining cavity drained.

Chloral, 1 gr., was given six-hourly for three doses, and oxygen continuously. The right saphenous vein was cut down upon and $250 \mathrm{ml}$. blood given in five hours; saline, $150 \mathrm{ml}$., with hyalase was injected subcutaneously to follow the blood.

On April 6, 1950, the general condition was satisfactory. The infant was well hydrated, and passing urine normally. There was some distension of the abdomen. Small amounts of $\frac{1}{2}$ normal saline and $5 \%$ 'casydrol ' were given by mouth. Vitamin C, $500 \mathrm{mg}$., was given intramuscularly. A course of heparin, 5,000 units twice daily, was begun.

On April 7, 1950, the general condition was good. The baby passed a fatty stool. The abdomen was soft, and not distended. The next day, April 8, the drainage tube was removed satisfactorily. On April 9 there was a small haemorrhage from the wound. The patient's condition was satisfactory. The heparin was stopped. The baby's condition improved, and he was taking cow's milk from April 6, without any vomiting. He subsequently developed infection of the wound, which was proved on culture to be due to Bact.coli as in the original urinary infection. The wound infection delayed healing, but the general condition of the baby slowly improved.

By May 15, 1950, the wound was completely healed, but there was weakness in the underlying muscles due to the infection which caused a separation of the sutured muscles.

The baby was discharged from hospital on May 17, 1950, when his condition was quite satisfactory. He had steadily gained weight until on discharge he weighed $9 \mathrm{lb} .10 \mathrm{oz}$.
He was seen in the out-patient clinic on May 31, 1950, when progress had been steady, and his weight was $11 \mathrm{lb} .6 \mathrm{oz}$.

Pathological Report. The macroscopic appearance of the left kidney showed enlargement with well marked foetal lobulation. Beneath the capsule there were confluent areas of deep congestion, alternating with pale grey areas. The cortex and medulla appeared to merge in a uniform dark haemorrhagic mass, although at some points the differentiation could be clearly seen. The calyces were dilated and contained a mass of soft grey tissue which arbourized at the upper and lower pole. The renal vein contained a thrombus.

The microscopic appearance showed absolute infarction of the kidney substance by a septic thrombus travelling through branches of the renal vein (Fig. 1). There was widespread inflammatory change throughout the infarcted area.

\section{Discussion}

Renal vein thrombosis is a rare condition and unilateral cases are still more rare. The condition should always be borne in mind when bloodstained urine is passed in the neonatal period. The presence of a renal swelling in neonatal life, particularly with infected urine, must also bring renal vein thrombosis into the differential diagnosis.

Predisposing factors of vomiting and infection are of extreme importance in making the diagnosis, though it is said that the condition can occur when these are absent. The predisposing factor is then said to be a condition of asphyxia with resulting general venous congestion and stagnation (Fallon, 1949).

Since most cases of renal vein thrombosis are diagnosed in the necropsy room, there is no uniformity of opinion regarding treatment.

Bilateral renal vein thrombosis usually terminates fatally, and surgery has no place in its treatment. Anti-coagulant therapy with heparin and dicoumarol has not yet been reported.

The role of surgery in the treatment of unilateral cases is of importance. Campbell and Matthews (1942) describe two cases of unilateral thrombosis treated surgically. They also advocate administration of vitamin $\mathrm{K}$ because of a low prothrombin level and haematuria. Administration of vitamin K, however, is absolutely contrary to the present conception of thrombosis and its treatment in general. In the case presented a low prothrombin level was not found: indeed, this was normal compared with a control. The risk of spreading thrombosis by administering vitamin $\mathrm{K}$ must be considerable and contra-indicates its use.

Nephrectomy in the unilateral case is not devoid of the risk that unsuspected thrombosis may have 
started on the other side. Nephrectomy, however, can be life-saving where the thrombosis, as in most cases, is infective in origin and manifested by heavily infected -urine. Fallon (1949) suggests this as the indication for nephrectomy.

The post-operative administration of heparin following nephrectomy may be of value in preventing thrombosis from occurring in the remaining kidney, or in arresting early thrombosis which may be present at the time of operation and impossible to detect by examination of the kidney at operation.
Summary

A classical case of unilateral renal vein thrombosis with treatment by nephrectomy and post-operative heparin is described.

The aetiology is briefly chronicled.

The treatment, particularly the role of surgery, is discussed.

Abeshouse, B. S. (1945). Urol. cutan. Rev., 49, 661.

Campbell, M. F., and Matthews, W. F. (1942). J. Pediat., 20, 604.

Fallon, M. L. (1949). Archives of Disease in Childhood, $24,125$. 